Meta

Journal des tradlucteurs

Translators' Journal

\title{
Table ronde sur le service de traduction dans l'entreprise
}

\section{Pierre Marchand}

Volume 21, numéro 1, mars 1976

La traduction et l'entreprise

URI : https://id.erudit.org/iderudit/003561ar

DOI : https://doi.org/10.7202/003561ar

Aller au sommaire du numéro

Éditeur(s)

Les Presses de l'Université de Montréal

ISSN

0026-0452 (imprimé)

1492-1421 (numérique)

Découvrir la revue

Citer cet article

Marchand, P. (1976). Table ronde sur le service de traduction dans l'entreprise. Meta, 21(1), 27-41. https://doi.org/10.7202/003561ar d'utilisation que vous pouvez consulter en ligne.

https://apropos.erudit.org/fr/usagers/politique-dutilisation/ 


\section{Table ronde sur le service de traduction dans l'entreprise}

Meta a jugé intéressant de demander à des traducteurs expérimentés de se réunir afin de discuter librement de l'organisation du service de traduction dans l'entreprise. Comme les lecteurs peuvent le constater, les participants ont naturellement fait appel à leur propre expérience afin de réaliser l'objectif de la table ronde : imaginer ce que, dans l'idéal, doit être le service de traduction.

Notre intention était de réunir six personnes provenant de milieux de travail différents ; trois d'entre elles ont effectivement accepté notre invitation et nous les en remercions chaleureusement. Il s'agit de M. André Caron, des Services linguistiques de la société Bell Canada, de M. Jean-Marc Lambert, rédacteur-traducteur à l'Hydro-Québec et de M. Gabriel Massy, directeur du service de Traduction de la Banque de Commerce Canadienne Impériale.

La discussion a porté sur sept sujets ayant comme point commun l'organisation concrète du service de Traduction. Il convient cependant de noter que les sujets ont été discutés indépendamment, ce qui explique la présentation du texte en sept parties apparemment sans lien. C'était, avons-nous pensé, la seule façon de présenter clairement le débat ; nous sommes convaincus que les lecteurs n'éprouveront pas de difficultés à établir eux-mêmes les liens et à tirer leurs conclusions, si différentes soient-elles.

Pierre Marchand

1. LE CHOIX DU DIRECTEUR ET LA PLACE

DU SERVICE DE TRADUCTION DANS L'ORGANIGRAMME DE L'ENTREPRISE

P. M. - Ce que j'aimerais que nous fassions ensemble, c'est d'essayer d'imaginer le service de Traduction idéal en supposant que nous avons tous les moyens à notre disposition et toute la latitude voulue. Dès le début, par conséquent, il faut créer le service et prévoir la façon dont on va en assurer la direction. Nous devons donc nous demander quel genre de personne il faut nommer comme chef de service. 
Il y a deux solutions possibles; certaines entreprises confient la direction du service à un traducteur d'expérience, qui connaît par conséquent le métier et les problèmes que peuvent éprouver les traducteurs. D'autres services songent plutôt à un administrateur, qui est censé connaître les questions d'administration et la façon de concevoir les relations entre les membres du personnel. $A$ votre avis, quelle solution faut-il choisir?

J.-M. L. - La solution que nous avons adoptée à l'Hydro-Québec fonctionne très bien. Chez nous, le chef de service consacre tout son temps à l'administration; il ne s'occupe pas des fonctions de révision ni de traduction. Il s'occupe de la bonne marche du service, il voit à lui procurer de la documentation et se charge d'établir et de maintenir les relations avec les autres directions de l'Hydro-Québec. À cause du noyau de rédacteurs-traducteurs très expérimentés qui sont là depuis les débuts, le chef de service s'intéresse moins aux préoccupations de langue; ce n'est pas qu'il les ignore cependant. Il s'occupe plutôt d'administrer, de veiller à ce que tout fonctionne bien ; c'est pourquoi, du point de vue matériel, le service ne manque de rien. Dans l'ensemble, cette solution nous a bien servis. Le fait d'avoir un traducteurréviseur comme chef de service n'est peut-être pas mauvais, mais chez nous, à partir de notre expérience, je crois qu'il est préférable que le chef de service soit un administrateur.

G. M. - Il faut dire que vous avez un service organisé depuis déjà fort longtemps...

J.-M. L. - Depuis une quinzaine d'années. Lorsque notre chef de service est entré dans l'entreprise, le service Rédaction et Traduction n'existait pas. La direction générale du Personnel lui a confié la rédaction de documents internes. Il a alors engagé quelques personnes pour effectuer ces travaux. Petit à petit, il est allé chercher des travaux à l'intérieur de l'entreprise en convainquant les autres services de la nécessité de rédiger des textes dans un français correct; le service a par conséquent pris de l'ampleur, c'est pourquoi le chef de service ne s'est plus consacré qu'à l'administration, à aller chercher les travaux, à faire la propagande du service.

P. M. - Et vous, M. Massy, qu'en pensez-vous ? Quels doivent être les états de service du chef de traduction?

G. M. - Moi je pense que c'est dans la mesure où l'effectif d'un service de traduction devient quantitativement important que le chef doit avoir des qualités d'administrateur. Par contre, s'il s'agit d'un petit service dont l'effectif ne dépasse pas les trois ou quatre traducteurs, le chef devra nécessairement être lui-même un bon traducteur afin de pouvoir réviser efficacement le travail de ses collègues et leur donner la formation voulue.

P. M. - À votre avis, par conséquent, les relations dans le service sont meilleures si celui-ci est dirigé par une personne de la même profession...

G. M. - J'en suis persuadé, et j'ajouterai que cela constituerait même un facteur de stimulation pour ses collègues, car si ces derniers sont convaincus de la compétence de leur chef dans le domaine qui les concerne, et du fait qu'il sera à 
même d'apprécier leur travail à sa juste valeur et, au besoin, de les dépanner, ils n'en seront que plus confiants et satisfaits.

A. C. - D'après mon expérience, le directeur du service devrait être ou aurait avantage à être traducteur, mais ce n'est peut-être pas essentiel. Il est bien évident qu'il peut y avoir des affrontements si le chef de service n'est pas un professionnel ou ne peut pas évaluer la qualité du travail. Mais dans un service assez important, il doit y avoir quand même des réviseurs, qui eux sont des professionnels pouvant évaluer le travail. À ce moment-là, les risques d'affrontement sont réduits de beaucoup. Quant aux risques de conflits avec les différents services-clients, là je pense qu'il y a avantage à ce que l'administrateur soit vraiment compétent et qu'il puisse prendre des positions fermes face aux demandes parfois exagérées. Je maintiens donc que le directeur du service aurait avantage à être un traducteur professionnel,

J.-M. L. - Je suis d'accord avec cela aussi. Chez nous par exemple, le chef de service est évidemment capable d'évaluer la qualité du travail, mais il est avant tout un administrateur. Ce sont les chefs de division qui s'occupent surtout des questions linguistiques.

G. M. - Sur le plan linguistique, le service de traduction a certes un rôle important à jouer dans l'entreprise. Il lui appartient, entre autres, de contribuer à l'amélioration du niveau de la langue dans le travail, et surtout de la langue écrite. Il doit aussi, spécialement dans les entreprises à direction anglaise au Québec, servir pour ainsi dire de trait d'union entre la direction anglaise et le personnel, qui est actuellement en majorité d'expression française.

P. M. - Alors, si l'entreprise est anglophone, il est encore plus important que le chef de service soit lui-même un traducteur, afin de mieux comprendre les questions de langue?

G. M. - Oui, bien sûr. Je voudrais aussi ajouter qu'il se présente parfois des cas où la direction de l'entreprise insiste, lorsqu'il s'agit de textes de caractère très confidentiel, pour que leur traduction ne soit confiée qu'à une seule personne et c'est souvent au chef de service lui-même qu'il est demandé de s'en charger. Il faut donc qu'il sache bien traduire pour pouvoir faire face à de telles éventualités.

P. M. - Après avoir trouvé un chef, il nous faut maintenant songer à loger le service de Traduction dans l'organigramme de l'entreprise. Certaines sociétés confient le service de Traduction au service du Personncl, d'autres à la Commercialisation, aux Relations publiques, à l'Expansion ou aux services les plus fantaisistes possibles. À votre avis, que doit-il être ?

A. C. - À Bell Canada, la situation en 1975 est sensiblement différente de ce qu'elle était il y a dix ans. À ce moment-là, il s'agissait surtout d'informer le public; les Relations publiques devaient faire traduire leurs textes et les besoins étaient considérables. Il allait donc de soi que le service de Traduction dépende des Relations publiques. Aujourd'hui, la situation est différente. Le personnel des entreprises est encouragé à travailler en français et les besoins se trouvent accrus. Il serait alors beaucoup plus logique que le service de Traduction dépende du Personnel à cause, entre autres, de la question de la formation. 
G. M. - À mon avis, le service de traduction d'une grande entreprise doit, dans le contexte actuel, relever principalement de la direction de l'entreprise, en raison de l'importance de plus en plus grande que l'on attache au fait français au Québec et à l'amélioration de la langue écrite dans les affaires.

P. M. - Jusqu'ici, nous avons donc deux points de vue aussi intéressants l'un que l'autre. Dans un cas, on préfère confier le service de Traduction au service du Personncl, car les traducteurs ont un certain rôle à jouer en matière de formation ; et, dans l'autre cas, on rapproche le service de Traduction de la haute direction, afin de faciliter le dialogue sur les grandes politiques linguistiques de l'entreprise. Et vous, M. Lambert, qu'en pensez-vous?

J.-M. L. - Chez nous, à l'Hydro-Québec, le service Rédaction et Traduction relève actuellement de la direction générale du Personnel, mais nous sommes en train de faire notre examen de conscience à ce sujet. Il n'est pas absolument certain que cette solution soit parfaite, car notre travail touche à tous les aspects de l'entreprise. Comme nous dépendons actuellement d'une direction générale, notre position n'est pas toujours très forte lorsqu'il faut travailler pour d'autres directions. $\mathrm{Si}$ notre service relevait plus directement de la haute direction, notre autorité morale s'en trouverait peut-être améliorée.

\section{LA RÉPARTITION DES TÃCHES}

P. M. - Nous allons maintenant nous tourner vers l'intérieur du service que nous avons à inventer. Prenons, pour commencer, le cas de la révision. Dans certains services, il y a des réviseurs attitrés; dans d'autres, ce sont les traducteurs qui se révisent eux-mêmes. Selon vous, quelle méthode est préférable?

J.-M. L. - Chez nous, nous avons toujours travaillé en équipe. Lorsqu'un rédacteur-traducteur a terminé son travail, il le fait réviser par un autre, de préférence le chef de division ou une personne plus ancienne dans le service. Le fait de ne pas avoir nécessairement un réviseur attitré nous permet des liens plus souples à l'intérieur du service ; c'est sûrement l'un des facteurs qui fait que l'atmosphère est très bonne chez nous.

G. M. - Chez nous, à cause du développement très rapide du service, dont l'effectif a plus que doublé depuis l'an dernier, il a fallu procéder à sa réorganisation et créer deux sections : une pour le travail général (traduction de circulaires, lettres, formules, etc.) et l'autre pour la traduction du manuel de la Banque. La consultation entre les traducteurs se fait aussi bien au sein de chaque section qu'entre une section et l'autre.

P. M. - La hiérarchisation n'est pas trop contraignante, les relations ne sont pas trop officielles?

G. M. - Non, pas du tout. Nous admettons, comme je l'ai déjà mentionné, que les traducteurs se consultent avant de remettre leurs textes à leurs réviseurs. On leur laisse d'autant plus de liberté dans ce domaine qu'aucun quota de production ne leur est imposé. 
A. C. - $\AA$ Bell Canada, le service existe depuis 25 ans. Jusqu'au printemps de 1975 , il était très structuré. Il y avait un directeur pour le service et un réviseur pour chacun de nos groupes de travail ; les réviseurs étaient des traducteurs chevronnés ; ils avaient la charge de leur groupe tant sur le plan administratif que sur le plan professionnel. C'était donc un véritable encadrement, qui avait ses avantages au point de vue de la formation : les réviseurs avaient des contacts quotidiens avec les traducteurs ; ceux-ci étaient donc suivis de très près. Le service évoluant, on en est venu à se demander s'il ne fallait pas plutôt former ce que nous appelons un «bassin de traduction». Nous avions réuni tout le service afin de repenser notre formule. À cette occasion, on a suggéré le décloisonnement du service pour permettre à chaque traducteur d'œuvrer dans tous les domaines. Cette méthode de travail a beaucoup d'avantages, mais elle présente des inconvénients en ce qui concerne la révision. Il y avait en effet des traducteurs qui étaient habitués à la relation supérieure-subalterne et qui voyaient d'un œil un peu suspect le fait de se faire réviser par quelqu'un d'un autre groupe, dont la compétence était reconnue, mais qui était leur égal.

G. M. - Il faut aussi veiller à assurer une certaine relation entre les diverses sections d'un service afin de susciter l'intérêt chez les traducteurs et d'éviter la monotonie.

P. M. - Vous abordez justement la question que je me proposais de vous soumettre et j'aimerais que vous me donniez plus de précisions sur l'opportunité de spécialiser ou non les traducteurs.

G. M. - Chez nous, comme la majeure partie du travail se rapporte en général aux domaines bancaire et financier, les traducteurs, après quelques années d'expérience, finissent par devenir en quelque sorte des spécialistes dans ces domaines. Il est assez rare, par exemple, que nous ayons à traduire des textes de caractère purement technique.

P. M. - Est-ce que chez vous, M. Caron, la spécialisation des travaux est plus apparente que dans le domaine bancaire, de sorte que vous ayez songé à répartir les tâches?

A. C. - Jusqu'au printemps dernier, la structure du service favorisait nécessairement la spécialisation des traducteurs. Mais depuis l'implantation du «bassin de traduction », les traducteurs sont appelés à traduire une plus grande variété de textes.

P. M. - Cela dépend donc de l'entreprise, on ne peut faire de loi générale sur la question de la spécialisation.

J.-M. L. - C'est cela. Chez nous, il y a deux divisions. L'une s'occupe du domaine administratif et l'autre, du domaine technique. Alors le personnel se spécialise, mais en ayant une vue assez large sur l'ensemble du travail. Dans la division technique, nous cherchons surtout à engager des personnes compétentes en rédaction et en traduction qui sont de plus spécialisées dans l'un des domaines techniques auxquels nous touchons. Dans la section administrative, il y a aussi des spécialistes, par exemple en comptabilité. 


\section{L'UTILISATION DES MOYENS TECHNIQUES DE TRANSCRIPTION}

P.M. - Si vous le voulez, nous allons maintenant passer à la question des moyens techniques à mettre ou à ne pas mettre entre les mains du traducteur. $M$. Massy, voulez-vous nous donner votre point de vuc sur l'utilisation du dictaphone?

G. M. - Il semblerait à première vue, surtout si l'on n'a jamais soi-même eu recours au dictaphone, que seul un traducteur chevronné puisse l'utiliser avec profit. Mais d'après les témoignages de personnes qui l'ont expérimentée, cette méthode de travail, tout en étant pratique et efficace, permet aussi d'atteindre un certain niveau d'uniformité entre le langage parlé et le langage écrit, ce qui se traduit naturellement par une amélioration appréciable de l'expression orale.

Je pense toutefois que lorsqu'il s'agit de traduction de textes techniques exigeant des recherches, l'usage du dictaphone devrait perdre beaucoup de son utilité.

A. C. - Je ne partage pas votre avis. Plusieurs de nos traducteurs en ont fait l'essai. On a même démontré qu'on peut traduire des formulaires au dictaphone, c'est une question de méthode. Lorsqu'on traduit, l'idéal ne serait-il pas de lire d'abord le texte afin de faire un relevé des termes présentant des difficultés, ensuite de faire les recherches nécessaires et enfin de se lancer dans la traduction ? Un de nos traducteurs a prouvé qu'un texte de 3000 mots et de difficulté moyenne exige une demi-journée de préparation pour environ une journée de dictée.

G. M. - Mais ce qui arrive très souvent un peu partout, c'est que beaucoup de textes à traduire sont de caractère très urgent, mettant ainsi les traducteurs dans l'impossibilité de réfléchir longuement ou de pousser leurs recherches. Il va sans dire qu'en pareilles circonstances, la vitesse va parfois au détriment de la qualité, peu importe que l'on utilise ou non le dictaphone.

A. C. - Il faut dire que cet essai n'a pas produit tous les résultats que nous en attendions. Même s'il a été démontré que la machine à dicter est un bon instrument de travail, il semble qu'on ne délaisse pas facilement la machine à écrire pour la machine à dicter.

G. M. - C'est possible, mais je pense que l'usage du dictaphone ou de la machine à écrire dans un service où les traducteurs ne sont séparés que par des cloisons insuffisamment insonorisées peut constituer un obstacle à la concentration et je me demande si les traducteurs seraient capables de travailler dans un tel vacarme.

J.-M. L. - Moi, je me suis habitué à travailler au rythme de la machine à écrire lorsque j'ai un texte à traduire et, chez nous, plusieurs autres rédacteurstraducteurs se servent de ce moyen. Il m'arrive même de récrire à la machine un texte que je suis à réviser et qui comporte trop de corrections. Tout cela, c'est une question d'habitude.

G. M. - Chez nous la question du travail à la machine a été définitivement tranchée, en ce sens que seules les secrétaires du service utilisent la machine pour dactylographier les textes que leur remettent les traducteurs. 
A. C. - On peut quand même écrire plus vite à la machine qu'à la main.

G. M. - Je n'en disconviens pas, bien que l'on arrive généralement avec un peu de pratique, à écrire assez vite. En tout cas, dans notre service, l'emploi de la machine ne serait supportable que si les traducteurs étaient séparés par des cloisons insonorisées.

A. C. - Chez nous, nous avons l'aménagement paysager et les gens ne s'en plaignent pas. Quoi qu'il en soit, je crois que le déblocage qui s'est produit à propos de la machine à écrire devrait également avoir lieu en ce qui concerne la machine à dicter. Peut-être pas chez les traducteurs plus âgés, mais chez les plus jeunes.

P. M. - Mais en ce qui concerne le principe même de la machine à dicter, certains se sont demandé si cette façon de traduire ne modifiait pas le style du texte rendu.

J.-M. L. - L'expérience dont j'ai été témoin était valable parce que ceux qui se servaient du dictaphone avaient à traduire un dialogue, c'est-à-dire les débats de la Chambre des Communes. Mais c'est probablement le seul endroit où j'ai eu l'impression que l'emploi du dictaphone pouvait bien fonctionner.

\section{LES FONCTIONS DU SERVICE DE TRADUCTION}

P. M. - Nous allons maintenant parler des fonctions du service de Traduction. L'on sait que certains services acceptent d'effectuer à peu près tous les travaux. D'autres auront plus de restrictions. Qu'en pensez-vous ?

J.-M. L. - Il y a quelques années, nous acceptions tous les textes, parce qu'il n'y en avait pas tellement. Aujourd'hui, ce n'est plus le cas. Nous avons établi trois critères qui déterminent les priorittés dans le choix des travaux du service : la diffusion, le caractère normatif et la portée du travail. De toute facon, nous sommes les seuls juges.

G. M. - Nous sommes censés accepter tous les textes qui nous parviennent de deux sources principales, lesquelles fixent aussi les priorités. Ces deux sources sont : le directeur de l'administration, à Montréal, et les secrétaires adjoints, à Toronto. En cas de chevauchement de priorités, nous communiquons immédiatement avec les intéressés pour mettre au clair la situation et nous attaquons alors en premier lieu les travaux qui ne souffrent pas de retard. D'autre part, lorsqu'un membre du personnel s'adresse directement à notre Service pour l'aider à mettre au point une lettre ou un texte rédigé en français et se rapportant au travail de la Banque, nous ne refuserons jamais de le faire.

P. M. - Et est-ce que vous avez établi, avec la direction, certaines normes de sélection des textes?

G. M. - Chez nous, la direction en a établi au moins une en ordonnant que, sous aucun prétexte, la traduction du Manuel de la Banque ne peut être retardée.

P. M. - Est-ce que c'est facile à faire ? 
G. M. - Ce n'est pas facile parce qu'il y a des jours où nous sommes vraiment débordés par toutes sortes de travaux urgents, et la détermination des priorités pose alors un vrai problème, car c'est à nous qu'il appartient le plus souvent, en pareil cas, de prendre les initiatives qui s'imposent.

P. M. - Est-ce que vous avez de la difficulté à respecter vos priorités ? Est-ce qu'il arrive que l'on vous fasse remettre à plus tard un travail urgent, afin de vous demander de traduire un autre travail urgent ?

G. M. - Oui, cela arrive parfois. Mais en général, à part le travail du Manuel qui ne doit jamais s'arrêter, c'est nous qui décidons en fin de compte de ce qu'il convient de terminer en premier lieu et je pense que c'est mieux ainsi.

A. C. - Chez nous, le service de Traduction a changé d'appellation; il s'appelle maintenant «Services linguistiques $»$. On a donc ouvert la porte à toutes sortes de demandes qui débordent tout à fait la traduction. J'ai l'impression que l'activité traduction va diminuer graduellement avec les années. Actuellement, l'activité terminologique est très importante et très structurée. Il y a aussi la consultation, l'aide à la rédaction. Nos tâches se diversifient donc considérablement. Quoi qu'il en soit, c'est nous qui déterminons les priorités, même lorsque le client passe par les échelons supérieurs de l'entreprise afin de nous faire accepter que l'on effectue son travail en priorité.

P. M. - Cette diversification des tâches du service de Traduction m'amène à vous demander si le rôle fondamental du service n'est pas appelé à se transformer et si, dans l'avenir, le service de Traduction s'occupera moins de traduction pour s'attacher davantage à la question globale de la francisation de l'entreprise.

G. M. - En effet, il est assez probable que plus la francisation progressera au sein des entreprises au Québec, moins les services de traduction auront à traduire vers le français du fait que la plupart des textes seront alors rédigés directement en cette langue.

P. M. - Ce qui est peut-être une façon, pour le service de Traduction, de se départir de l'excédent des travaux de traduction.

G. M. - Il se pourrait fort bien.

P. M. - Il faut constater, pour le moment, que le service de Traduction est une sorte d'entonnoir par lequel passent les demandes de tous les services de l'entreprise.

G. M. - C'est ce qui explique d'ailleurs l'importance que prend ce service au sein de l'entreprise. Il faut cependant s'attendre à ce qu'à mesure que progressera la francisation et que les employés prendront l'habitude d'écrire directement en français, la quantité des textes à traduire en cette langue tendra à diminuer. Par contre, il y aura davantage de textes à traduire vers l'anglais pour la simple raison qu'il faudra mettre les sièges sociaux des entreprises anglaises au courant des décisions qui seront alors prises en français.

J.-M. L. - Chez nous à l'Hydro-Québec, la fonction « rédaction » est déjà une réalité. 


\section{LA TERMINOLOGIE ET LA DOCUMENTATION}

P. M. - Passons maintenant à la question de la terminologie et de la documentation. À quel moment de son histoire, le service doit-il engager un terminologue?

A. C. - Moi je dirais dès le début et même avant. Si on me demandait de créer un service de traduction, je recommanderais que le service ne fasse pas de traduction durant les six premiers mois, voire même la première année de sa création. Il faudrait d'abord mettre au point la terminologie et constituer la documentation.

G. M. - Chez nous, étant donné que notre travail ne comporte pas beaucoup de recherches dans le domaine technique, nous n'avons vraiment pas eu besoin d'un terminologue jusqu'à présent. Les circonstances doivent cependant être bien différentes dans les sociétés où la majeure partie du travail exige des recherches dans ce domaine.

A. C. - Vous n'avez pas l'impression que vous y gagneriez quand même à avoir un terminologue?

G. M. - Nous y gagnerions sans aucun doute si les traducteurs ne manifestaient pas d'intérêt à faire eux-mêmes les recherches nécessaires. Or, chez nous ils les font et ils en consignent les résultats sur des fiches qui sont ensuite classées dans notre fichier central. C'est un peu pour cette raison que nous n'avons pas jugé nécessaire jusqu'à présent d'engager un terminologue.

A. C. - Eh bien! chez nous, nous avons deux terminologues et il y a de la recherche qui se fait constamment sur le plan technique.

G. M. - C'est justement parce qu'il s'agit de recherches sur le plan technique que c'est différent, car ces recherches exigent plus de temps et de spécialisation.

A. C. - Oui, j'admets que c'est différent. Chez nous, il y a constamment de nouveaux équipements mis en service et nos terminologues seraient occupés vingtquatre heures par jour.

J.-M. L. - Chez nous, on n'a pas officiellement de terminologue, quoique certains, dans le service, s'occupent un peu plus que les autres de terminologie. Il faut dire que la documentation est assez importante puisque nous avons 22 documentalistes. service?

A. C. - M. Massy, est-ce que vous avez un fichier à l'usage de tout le

G. M. - Oui, nous avons un fichier à l'usage du service, qui est continuellement mis à jour. Il contient actuellement quelque 6000 fiches et devrait en contenir presque le double une fois complété. Je dois ajouter que nous y incorporons les fiches de Radio-Canada, de même format que les nôtres.

A. C. - Imaginez si tous les travaux de terminologie étaient mis en commun !

J.-M. L. - Nous avions formé le projet d'envoyer des copies de nos fiches à la Régie de la langue française pour qu'elles soient intégrées dans sa banque de terminologie. Toutefois, le manque de temps ne nous a pas permis de le réaliser. 


\section{IE RECRUTEMENT}

P. M. - Passons maintenant à la question du recrutement. À votre avis, le service de Traduction doit-il engager des traducteurs qui n'ont pas d'expérience, afin de les former en cours de travail (de crainte qu'ils n'aient pris de mauvaises habitudes ailleurs) ou, au contraire, doit-on faire confiance à l'expérience que les gens ont acquise dans d'autres entreprises?

G. M. - C'est un peu difficile à préciser, car le facteur chance y est pour beaucoup. Ainsi, certains candidats qui passent bien l'examen d'admission ne donnent, une fois au travail, que peu de satisfaction quant au rendement et aux progrès auxquels on s'attendait de leur part. D'autres, par contre, sans avoir particulièrement brillé à l'examen à cause, peut-être, de l'émotion du moment, se révèlent par la suite de très bons traducteurs, appliqués et consciencieux. Par ailleurs, comme il est absolument nécessaire pour un traducteur de savoir bien rédiger, nous avons ajouté, à l'examen en traduction, une composition française sur un sujet laissé au choix du candidat, ce qui nous permet de juger de sa compétence en rédaction.

J.-M. L. - Chez nous, nous accordons beaucoup d'importance à la rédaction. De plus, nous exigeons généralement un diplôme en traduction. Pour ce qui est des travaux techniques, nous tentons de recruter des personnes qui ont fait des études dans le domaine concerné, mais ces candidats sont très rares. Nous avons récemment engagé des personnes qui terminaient leurs études de maîtrise en traduction et nous avons alors comparé leur examen avec ce qu'ont fait des gens censément plus expérimentés; nous nous sommes aperçus, en fin de compte, que les jeunes diplômés réussissaient très bien.

G. M. - Je voudrais ajouter que certains traducteurs montrent d'excellentes aptitudes en cours d'emploi tandis que d'autres, après un stage de six mois ou même d'une année, en sont encore presque au même point. C'est donc une question d'aptitudes personnelles. Il faut aussi que le traducteur aime son métier, autrement il n'y aurait pas grand-chose à en tirer. D'autre part, la formation de base en français est essentielle au traducteur s'il veut exceller dans son métier.

J.-M. L. - Chez nous d'ailleurs, on préférera quelqu'un qui a une bonne connaissance du français plutôt qu'une personne qui a d'excellentes connaissances techniques mais qui rédige dans un français de moins bonne qualité.

G. M. - C'est un peu mon principe aussi.

P.M. - Est-ce que cette formation en français, on peut aller la chercher ailleurs qu'à l'université ou à l'école de traduction ?

G. M. - Si le candidat (sans être diplômé en traduction) a une formation en français telle qu'elle lui permet de passer honorablement l'examen, je pense qu'on devrait lui donner une chance, surtout s'il a déjà à son actif une certaine expérience comme pigiste ou comme rédacteur.

J.-M. L. - Si l'étudiant qui arrive de l'école de traduction possède une bonne connaissance du français, il n'aura aucune difficulté. C'est pourquoi l'examen que nous faisons subir aux candidats est conçu de façon à nous permettre d'évaluer leurs connaissances de base. 
G. M. - L'entrevue qui suit l'examen a également son importance, car elle permet de connaitre un peu la personnalité du candidat, ses aspirations et le penchant qu'il manifeste pour le genre de travail que l'on compte lui confier.

A. C. - Notre meilleure source de recrutement nous est fournie par les stages. Chaque été, nous embauchons trois ou quatre étudiants de deuxième année de traduction à l'Université de Montréal. De ces stagiaires, il y en a presque toujours un qui émerge et représente le candidat idéal.

P. M. - Est-ce que les stagiaires font un travail utilisé par le service ?

A. C. - Ah oui! dès le début. On en est venu à la conclusion qu'il valait mieux mettre les stagiaires dans le bain dès la première journée; ils ont tout de suite des échéances à respecter.

P.M. - Le stage représente alors une sorte de période de formation?

A. C. - Très certainement ; cette façon de faire est aussi avantageuse pour le stagiaire que pour le service.

P. M. - Cette question du recrutement m'amène à vous demander si le service de traduction ne doit pas, à un moment donné de son histoire, stopper sa croissance.

A. C. - Chez nous, on a établi le nombre de traducteurs qu'un réviseur peut avoir et le rapport est d'un réviseur pour trois traducteurs. Quant au nombre optimal de traducteurs dans un service, la question ne s'est pas posée.

G. M. - Chez nous, comme je l'ai déjà mentionné, le développement rapide du service nous a amenés à y réorganiser la répartition des tâches. Nous nous en tenons, pour le moment, à la proportion d'un réviseur pour trois traducteurs et les résultats sont satisfaisants.

A. C. - Il y a toute une partie de la question que l'on néglige, celle de la femme au travail. Nombre de traductrices s'accommoderaient fort bien de travailler à temps partiel. Pour elles, ce serait avantageux et les entreprises pourraient également y trouver leur compte. Certaines pourraient même travailler en soirée.

J.-M. L. - À condition, cependant, que la personne qui travaille en soirée puisse avoir le moyen d'entrer en contact avec ses informateurs.

A. C. - Oui, bien sûr. Quoi qu'il en soit, je crois que l'horaire fixe est un obstacle. On devrait de plus en plus se tourner vers l'horaire variable ; cela permettrait à un plus grand nombre de personnes de se servir des mêmes locaux.

G. M. - Oui, mais cela reste très relatif. Il m'est difficile de concevoir, par exemple, qu'un traducteur salarié ait la liberté, en vertu de l'horaire flexible, d'arriver au bureau à 6 heures de l'après-midi pour y travailler jusqu'à minuit, car s'il a déjà - chose plus que probable - déployé quelque activité durant la journée, son rendement à une heure aussi tardive ne pourra être vraiment efficace. Lorsqu'il y a un si grand décalage d'horaire, on ne peut s'empêcher d'être sceptique, aussi bien en ce qui a trait au rendement qu'à la qualité du travail fourni.

J.-M. L. - Chez nous, l'expérience de l'horaire variable avec place fixe n'a pas posé de gros problèmes; nous n'avions pas vraiment prévu un contrôle rigide. 
On fixait à l'avance un nombre d'heures à faire et chacun comptait ses heures. Cela a bien fonctionné.

A. C. - Nous avons l'horaire variable et cela ne pose pas de problèmes particuliers; il y a même certains avantages puisque, souvent, il y a des travaux à faire à la dernière minute. Vu que certains traducteurs quittent plus tard, il est possible de faire traduire les textes urgents sans devoir tenir compte des heures supplémentaires.

P. M. - Car les heures supplémentaires sont rémunérées chez vous ?

A. C. - Oui.

J.-M. L. - Chez nous aussi.

G. M. - Chez nous, on n'a pas encore commencé à faire des heures supplémentaires, mais lorsque les circonstances l'exigeront, cela se fera, avec rémunération bien entendu.

A. C. - M. Lambert, est-ce que les traducteurs et les rédacteurs sont syndiqués à l'Hydro-Québec ?

J.-M. L. - Non, ils ne le sont pas parce que notre service fait partie de la direction générale du Personnel. À cause du caractère confidentiel de l'activité de cette direction générale, aucun de ses employés n'est syndiqué.

P. M. - La question de la croissance du service nous a menés par bien des chemins ; j'aimerais, si vous le voulez bien, que l'on s'attarde un peu plus à l'importance quantitative du service. Certains services de traduction comptent plusieurs dizaines de traducteurs. À votre avis, est-il possible qu'un service fonctionne avec un personnel si important ?

G. M. - Il devrait bien fonctionner s'il était ogarnisé de la même façon que l'est toute grande entreprise, où les fonctions de chacun sont bien définies et où est appliqué le système hiérarchique entre les membres du personnel. C'est justement dans des cas comme celui-ci que la présence d'un bon administrateur est indispensable.

P. M. - Dans certains services quantitativement importants, le traducteur ne communique pas avec son réviseur; il reçoit en fin de mois son texte révisé.

G. M. - Cette méthode de travail, qui semble admise dans les très gros services, ne saurait donner de bons résultats dans des services de 10 à 15 traducteurs, où tout le monde travaille ensemble. Une constante communication entre le traducteur et son réviseur devrait donner, si elle se fait dans un esprit de bonne coopération, des résultats très constructifs.

P. M. - Est-ce que ce n'est pas la tentation des services géants de ne permettre que les relations dans un sens, c'est-à-dire du traducteur vers le réviseur vers le chef de service?

G. M. - Malheureusement oui, parce que cet état de choses ne favorise pas l'esprit d'équipe que l'on trouve dans les services de moindre importance et qui aide à créer une atmosphère de coopération très propice au travail. 
A. C. - Chez nous, nous avons cette année fait circuler parmi les réviseurs une photocopie de brouillons révisés de certains textes. De la sorte, l'ensemble des réviseurs, et le directeur du service, ont pu avoir une idée du travail qui s'est fait. C'est essentiel pour que le traducteur soit évalué le plus objectivement possible. Et notre méthode nous permet de diriger vers les traducteurs notre opinion sur leur travail. Quoi qu'il en soit, en principe, les grands services de traduction peuvent fonctionner, pourvu que les relations de travail soient fréquentes sinon quotidiennes et non pas mensuelles ou annuelles.

\section{LE RECOURS AUX TRADUCTEURS PIGISTES}

P. M. - Doit-on systématiquement recourir aux traducteurs-pigistes pour faire effectuer certains travaux précis, toujours les mêmes (par ex.: le rapport annuel) ou, au contraire, doit-on attendre que les cas urgents se présentent?

G. M. - Chez nous, on n'a recours à un pigiste que pour collaborer à la traduction du rapport annuel de la Banque ou encore, mais très rarement, pour la traduction de la publication mensuelle de la Banque.

P. M. - Mais si le service ne peut jamais répondre à toutes les demandes, que doit-il faire?

G. M. - Ou faire faire des heures supplémentaires aux traducteurs, ou augmenter l'effectif du service ou, si aucune de ces deux éventualités n'est réalisable, recourir alors aux services d'un pigiste.

P. M. - Serait-il alors souhaitable que le service de Traduction adopte la solution du pigiste permanent, presque toujours prêt à accepter l'excédent de travail?

G. M. - Ce serait très intéressant si nous pouvions lui garantir un volume assez régulier de travail, mais ce n'est pas encore le cas chez nous.

P. M. - Vous ne voudriez pas cependant confier, à l'avance, tel genre de texte à un pigiste ?

G. M. - On sera finalement obligé de le faire si le volume de travail continue d'augmenter et qu'il n'y ait plus aucune possibilité, pour des raisons d'ordre administratif, d'engager du personnel supplémentaire.

A. C. - Chez nous, c'est, entre autres, le caractère confidentiel ou non du texte qui détermine si l'on aura recours au traducteur pigiste. On envoie plutôt à l'extérieur des textes techniques.

\section{L'ÉVALUATION DU TRAVAIL}

P. M. - Nous allons en dernier lieu parler d'une question importante, l'évaluation du traducteur. Certains services accordent une grande importance à la question du rendement, qu'ils évaluent selon le nombre de mots traduits pendant une période donnée. D'autres services se diront que ce qui compte, c'est que le travail soit bien fait. $\mathrm{Y}$ a-t-il un juste milieu entre ces deux attitudes? 
A. C. - C'est une question de motivation et de responsabilité. Si le service est organisé de façon à ce que les traducteurs s'intéressent à leur travail, si les textes eux-mêmes sont, dans la mesure du possible, intéressants à traduire, le rendement quantitatif s'améliorera nécessairement.

J.-M. L. - Il faut aussi que le travail soit bien réparti.

G. M. - En fait, les deux facteurs sont importants : la qualité et le rendement. Si, au début, le rendement n'est pas suffisant, c'est parce que le traducteur n'a pas encore acquis assez d'expérience, mais s'il a les qualités requises, alors il faut s'attendre à ce que son rendement augmente naturellement avec le temps.

P. M. - Est-il réaliste de fixer une norme de rendement (de 1000 ou 1500 mots par jour) ?

G. M. - Oui, si le travail est uniforme. Dans ce cas, le traducteur peut même arriver à doubler son rendement initial. Mais si les textes sont très variés et exigent beaucoup de recherches, il semble qu'il soit assez difficile de pouvoir fixer une moyenne. C'est alors au superviseur ou au chef de service qu'il appartiendra d'apprécier la situation. Chez nous, bien que les textes à traduire soient assez variés, nous nous attendons à ce que le rendement et la qualité augmentent de 10 à 15 pour cent par année.

A. C. - Je ne dirais pas que la norme soit de 1000 ou 1200 ou 1500 mots par jour, mais on tient compte du nombre de mots traduits chaque jour pour l'établissement des moyennes individuelles et des objectifs mensuels.

P. M. - Est-ce que vous tenez compte de la difficulté des textes ?

A. C. - Nous nous sommes servis d'indices de pondération pendant un certain temps; puis nous sommes revenus au compte du nombre de mots réels. La technique de pondération était trop complexe ou mal appliquée.

P. M. - Comment faites-vous la différence entre la traduction d'un texte de 25000 mots et celle d'une vingtaine de formulaires confiés au même traducteur?

A. C. - L'on s'est rendu compte, en fait, qu'à force de traduire des textes techniques, ceux-ci devenaient graducllement aussi faciles que des textes courants. J'admets que les formulaires représentent un cas particulier; à venir jusqu'à tout récemment, l'évaluation était basée sur le nombre d'heures consacrées à la traduction des formulaires; ce nombre d'heures était converti en nombre de mots. Mais, en règle générale, tous les textes sont aujourd'hui sur le même pied en ce qui concerne le degré de difficulté. Personnellement, j'admets qu'il devrait y avoir un peu plus de nuances; il y a une différence entre traduire un rapport annuel et traduire un document technique. Il est d'autre part difficile d'établir le degré de « technicité » d'un texte.

G. M. - Il faut aussi tenir compte de la bonne volonté du traducteur et de son comportement vis-à-vis du travail. S'il fait tout son possible et fournit de bonnes traductions n'exigeant qu'une légère révision, il méritera d'être encouragé même si son rendement est légèrement inférieur à la moyenne générale du service. Il est également nécessaire, pour assurer un bon rendement dans tout le service, de veiller au maintien d'une bonne harmonie entre les traducteurs et leurs réviseurs. 
A. C. - Certains craignent qu'en faisant travailler ensemble des gens qui s'entendent trop bien, il y a risque que les révisions soient effectuées de façon trop indulgente.

G. M. - Ça dépend beaucoup de l'état d'esprit du traducteur. S'il est perfectionniste, il exigera de son réviseur qu'il lui corrige sérieusement ses textes, car c'est surtout de cette façon-là qu'il pourra améliorer son rendement et la qualité de son travail. Il y a aussi le contrôle général du travail par les responsables du service qui finiront bien, s'il y a négligence de la part de leurs collaborateurs, par s'en apercevoir.

P. M. - $\grave{A}$ votre avis, est-ce que les services de Traduction qui n'ont pas recours à l'évaluation quantitative commettent une erreur ?

G. M. - Pas nécessairement, parce qu'il faut aussi tenir compte de l'évaluation qualitative. Pour ma part, je sacrifierais volontiers sur la quantité si cela devait se traduire par un travail de meilleure qualité.

A.C. - Je réponds « oui » à votre question, mais l'évaluation quantitative n'est qu'un des éléments à considérer.

P.M. - D'autre part, puisque nous en sommes au rendement, j'aimerais savoir si, à votre avis, il est difficile de faire comprendre à l'entreprise que le rendement des traducteurs ne doit pas dépasser une certaine limite.

G. M. - Actuellement ce n'est pas difficile, surtout si des moyennes ont déjà été établies et acceptées. On peut même arriver à convaincre l'entreprise que plus les textes à traduire exigent recherches et consultations, moins on peut imposer un quota de production.

A. C. - Nous avons essayé d'éduquer nos clients afin de les amener à prévoir un temps raisonnable pour la traduction. C'est difficile, car il arrive encore qu'on nous demande de traduire en quelques jours un texte dont la rédaction anglaise a pris des semaines ou même des mois.

J.-M. L. - Il nous arrive assez fréquemment d'affecter un rédacteur à un service ou à un groupe d'étude qui prévoit avoir un travail de longue haleine à effectuer. C'est une façon de régler le problème.

P. M. - Au cours de la discussion de notre dernier sujet, vous nous avez donné une bonne idée de cc que devrait être le traducteur pour l'entreprise. Il nous resterait peut-être à demander aux traducteurs ce qu'est, pour eux, une bonne entreprise...

G. M. - ... et un bon directeur ! 